\title{
Comparative Evaluation of Different Mel-Frequency Cepstral Coefficient Features on Qura'nic-Arabic Verification
}

\author{
Fatma Khaled \\ Department of Systems \& Biomedical Engineering \\ Cairo University \\ Giza, Egypt
}

\author{
Sahar Fawzy \\ Department of Systems \& Biomedical Engineering \\ Cairo University \\ Giza, Egypt
}

\begin{abstract}
This research is concerned with facilitating the use of $\mathrm{Al}$ Qaeda Al Norania as an important Learning method in pronunciation proficiency of Arabic Language and Holy Qur'an. Speech Verification techniques were applied to detect how close the trainee's pronunciation is to the guaranteed reference reader. The impact of the number of coefficients and parameterization of Mel-Frequency Cepstral Coefficients (MFCC) on verification accuracy was tested and compared. Al Qaeda Al Norania records and HTK toolbox were used to generate the best verification model for selected Arabic Syllable.
\end{abstract}

\section{General Terms}

Hidden Markov Models (HMM), Mel-Frequency Cepstral Coefficient (MFCC), Arabic Speech Verification, HTK Toolbox .

\section{INTRODUCTION}

Arabic ranks sixth in the world's league table of languages, with an estimated 186 million native speaker [1]. Arabic usually designates one of three main variants: the language of Qur'an (Classical Arabic - CA), an adapted form of CA (Modern Standard Arabic - MSA), and Local dialects which is everyday spoken language that have many national or regional varieties [2].

Al Qaeda Al Norania was developed by Sheikh Nour Mohamed Hakany to teach the right pronunciation of Arabic phonemes. It is composed of 16 lessons, each of them introduces at least one pronunciation rule. Lessons of $\mathrm{Al}$ Qaeda Al Norania are very suitable for children and nonnative Arabic readers [3,4].

The majority of previous research concerning Automatic Arabic Speech Recognition and Verification focused on MSA, rather than CA [5]. Arabic syllables are clustered according to their constituents Exits or Characteristics. Exit is the exact position of the articulators organs from which phoneme is uttered and be differentiated from others. Letter characteristics depend on the vocal tract configuration, the exit and the vocal folds vibrations. Letters uttered from the same exit, differ in their characteristics while those sharing the same characteristics uttered from different exits.

In this study, models for clusters of CA Arabic syllables extracted from el Qaeda Al Norania records were investigated. These models were used to verify the trainee utterances.

The Verification task is implemented through two main steps:

\subsection{Feature Extraction}

Is the process of converting speech signal into limited set of parameters. One of the most commonly used feature extraction methods is Mel-Frequency Cepstral Coefficients (MFCC).

\subsection{Pattern Recognition}

Pattern Recognition is the task of finding a parameter set from references which closely matches the parameter set obtained from the input speech signal.

\section{RELATED WORK}

Points of research in Arabic language are still in progress. The majority of research studies were concerned with MSA while those concerning CA were limited.

In a recent work, Razak et. al. [6] have investigated Quraanic verse recitation feature extraction using the Mel-Frequency Cepstral Coefficient (MFCC) approach. Their paper explored the viability of the MFCC technique to extract features from Quranic verse recitation as it implements the DFT and FFT algorithm. Features extraction is crucial to prepare data for the classification process. H. Tabbal, et al [7] were interested in identifying the Qur'an Chanter accurately and reached accuracy up to $92 \%$, the experiments were limited to small Qur'an chapter.

Bassam et al. [8] developed \& implemented an Arabic Automatic Speech Recognition engine for both continuous speech and isolated words; Using MFCC and HTK. the overall system performance was $90.62 \%, 98.01 \%$ and $97.99 \%$ for sentence correction, word correction and word accuracy respectively. Vimala.C [9] compared the performance of the ASR system based on the adopted feature extraction technique and the speech recognition approach for the particular language; Based on the review, it was found that HMM approach along with MFCC features offers good recognition result for large vocabulary speaker independent continuous speech.

\section{METHOD}

Our methodology follow a sequence of phases, which are: Preparing the used Dataset, Feature Extraction, Acoustic Training and Pattern Recognition.

\subsection{Our Dataset}

Syllable is the basic unit in constituting a sequence of speech sounds. It is considered the phonological building block of words. Syllables consist of phonemes; where phoneme is the smallest speech unit that makes a difference in the meaning of a word, or a sentence [10]. Phonemes can be either consonants (C), vowels (V) or long vowels (V:).

Arabic Language has two types of syllable: open syllables $(\mathrm{CV})$ and $(\mathrm{CV}:)$, and closed syllables $(\mathrm{CVC}),(\mathrm{CV}: \mathrm{C})$ and (CVCC) [11]. 
Our data set components were collected from the second lesson of Al Qaeda Al Norania; in which Arabic consonants are combined in short syllables with only one vowel fathah, for example ( $\omega)$. The selected syllables were located in different co-articulation positions, as the first, middle or terminating syllable.

Analog signals for Arabic syllables are sampled at $22 \mathrm{KHz}$ and recorded by two guaranteed Readers of Holy Qur'an and trainers of Al Qaeda Al Norania; "Farok Al Ra'y" and "Osama Kari".

Table 1 shows syllables having same exits with their given corresponding IDs, and Table 2 shows syllables having similar characteristics with their given corresponding IDs as well.

Table 1. Syllables sets having same Exits

\begin{tabular}{|c|c|}
\hline Set ID & Syllable Sets \\
\hline ES1 & TTAA, DAAL, TAA \\
\hline ES2 & SEEN, SAAD, ZAA \\
\hline ES3 & ZAA, ZAAL, THAA \\
\hline ES4 & DDAAD, LAAM \\
\hline ES5 & NOON, RAA \\
\hline ES6 & GEEM, SHEEN, YAA \\
\hline ES7 & QAF, KAAF \\
\hline ES8 & 3AIN, 7AA \\
\hline ES9 & FAA \\
\hline ES10 & WAW, BAA, MEEM \\
\hline
\end{tabular}

Table 2. Syllables sets having same Characteristics

\begin{tabular}{|c|c|}
\hline Set ID & Syllable Sets \\
\hline CS1 & LAAM, NOON, 3AIN, MEEM, RAA \\
\hline CS2 & SAAD, DDAAD, TTAA, ZZAA \\
\hline CS3 & BAA, TTAA, QAF, GEEM, DAAL \\
\hline CS4 & BAA, TTAA, QAF, GEEM, DAAL, KAAF, \\
HAMZA, TAA
\end{tabular}

\subsection{Feature Extraction}

Different combinations of MFCC and its derivatives were applied in this work.

Sequences of feature vectors were extracted from speech waveforms in form of Mel-Frequency Cepstral Coefficients (MFCC). Our computational algorithm of the MFCC parameterization is obtained by a bank of symmetric overlapping triangular filters spaced linearly in a melfrequency axis, according to auditory perceptual considerations. The spacing as well as bandwidth of the particular filters is determined by a constant mel-frequency interval. In our case this feature extraction has been done with pre-emphasis coefficients 0.97 , the frame size was approximately 25 msecs window time for hamming window, and 10 msecs as window step size.

Speech input in HTK is controlled by configuration parameters. The key parameters are SOURCEKIND and
TARGETKIND which specify the source and target parameter kinds; that determine the end-points of the required input conversion [12]. In this research SOURCEKIND was raw data in form of wav files. Concerning TARGETKIND, two types (which had the highest correctness and Accuracy in an HTK study [13]), have been investigated: MFCC_0_D_A_Z and MFCC_E_D_A_Z. Each of these investigated target kinds have three different feature extraction schemes based on Mel Frequency Cepstral Coefficients (MFCC). The first scheme used 12 MFCC coefficients in a 39-dimensional feature vector which comprises also 12 delta coefficients, 12 acceleration coefficients and zeroth coefficient). The second scheme used 8 MFCC coefficients with its derivatives that resulted in 27dimensional feature vectors. The third scheme used 6 MFCC coefficients and resulted in a feature vector with 21 components.

\subsection{Acoustic Training}

Basic models of vocabulary syllables and a model for silence are included [14]. For each input utterance, a transcription is needed i.e. a list of the phones in that utterance. HTK then joins together all of the sub-syllable HMMs corresponding to this phone list to make a single composite HMM; this reference transcription file is to be used in HTK statistics.

Training of HMM models is done by applying Baum Welch algorithm on the training files provided. The algorithm requires specifying the number of states $\mathrm{n}$ of the learnt model (Our HMM models are belt with 11 states per each). The algorithm finds a local maximum in the parameter space of $n$ state HMMs, rather than a global maximum.

\subsection{Pattern Recognition}

HTK Toolkit was used for building and manipulating Hidden Markov Models (HMMs) used for utterances verification [15].

For training the HMM, HTK is used for estimating the optimal values for the HMM parameters (transition probability, mean and variance vectors for each observation function) in an iterative procedure known as re-estimation using the data from the speech files; and this is repeated several times for each HMM to train [16].

HTK is a Software Toolkit, It is an open source set of modules written in ANSI C developed by the Cambridge University (Young et al., 2011); we use it for both tasks feature extraction and pattern recognition.

The second step is pattern recognition task by means of HMMs testing. HTK Toolkit uses Viterbi-based speech recognition algorithm. The Viterbi Algorithm (VA) is often looked upon as minimizing the error probability by comparing the likelihoods of a set of possible state transitions that can occur, and deciding which of these has the highest probability of occurrence. The VA can be simply described as an algorithm which finds the most likely path i.e. shortest path, given a set of observations [17].

\section{RESULTS}

In the statistics file of HTK Toolbox, the sentence-level accuracy based on the total number of transcriptions generated by the recognizer which are identical according to the reference transcriptions. Secondly we get the word accuracy of the transcriptions generated by the recognizer [12]

The percentage of correctly recognized words is given by equation (1), the accuracy is computed by equation (2), and the word error rate is computer by equation (3). 
$\%$ Correct $=(\mathrm{H} / \mathrm{N}) * 100 \%$

$\%$ Accuracy $=((\mathrm{N}-\mathrm{D}-\mathrm{S}-\mathrm{I}) / \mathrm{N}) * 100 \%$

Word Error Rate $($ WER $)=1-\%$ Accuracy

Where, $\mathrm{H}$ is the number of correct words, $\mathrm{D}$ is the number of deletions (words that are present in the reference transcription, but are 'deleted' by the recognizer and do not occur in the recognizer's transcription), $\mathrm{S}$ is the number of substitutions (words in the reference transcription that are 'substituted' by other words in the recognizer's transcription), I is the number of insertions (words that are present in the recognizer's ranscription but not in the reference), and $\mathrm{N}$ is the total number of words in the reference transcription [12].

The results of all speech recognition experiments are summarized as follows: Results for Syllables having same exits with TARGETKIND "MFCC_0_D_A_Z" in Table 3 and Figure 1.

Table 3. Speech Verification experiments results for Syllables having same Exits with TARGETKIND MFCC_0_D_A_Z

\begin{tabular}{|c|c|c|c|}
\hline Set ID & Scheme & $\%$ Corr & $\%$ Acc \\
\hline \multirow{3}{*}{ ES1 } & 1 & 87.50 & 94.44 \\
\hline & 2 & 91.67 & 95.83 \\
\hline & 3 & 79.17 & 93.06 \\
\hline \multirow{3}{*}{ ES2 } & 1 & 100 & 100 \\
\hline & 2 & 100 & 100 \\
\hline & 3 & 100 & 100 \\
\hline \multirow{3}{*}{ ES3 } & 1 & 88.89 & 96.30 \\
\hline & 2 & 100 & 100 \\
\hline & 3 & 100 & 100 \\
\hline \multirow{3}{*}{ ES4 } & 1 & 100 & 100 \\
\hline & 2 & 100 & 100 \\
\hline & 3 & 100 & 100 \\
\hline \multirow{3}{*}{ ES5 } & 1 & 86.67 & 95.56 \\
\hline & 2 & 73.33 & 91.11 \\
\hline & 3 & 80.00 & 93.33 \\
\hline \multirow{3}{*}{ ES6 } & 1 & 89.47 & 96.49 \\
\hline & 2 & 89.47 & 96.49 \\
\hline & 3 & 84.21 & 94.74 \\
\hline \multirow{3}{*}{ ES7 } & 1 & 100 & 100 \\
\hline & 2 & 100 & 100 \\
\hline & 3 & 100 & 100 \\
\hline \multirow{3}{*}{ ES8 } & 1 & 87.50 & 96 \\
\hline & 2 & 100 & 100 \\
\hline & 3 & 100 & 100 \\
\hline \multirow{3}{*}{ ES9 } & 1 & 66.67 & 90 \\
\hline & 2 & 66.67 & 90 \\
\hline & 3 & 75 & 92.31 \\
\hline \multirow{3}{*}{ ES10 } & 1 & 100 & 100 \\
\hline & 2 & 100 & 100 \\
\hline & 3 & 100 & 100 \\
\hline
\end{tabular}

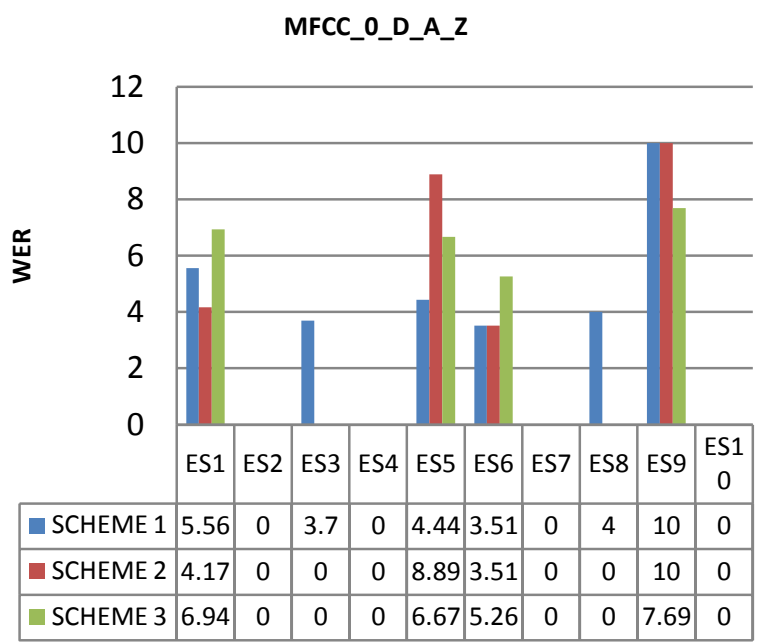

Fig 1: WER Results for Syllables having same Exits with TARGETKIND MFCC_O_D_A_Z

Results for Syllables having same exits with TARGETKIND "MFCC_E_D_A_Z" in Table 4 and Figure2.

Table 4. Speech Verification experiments results for Syllables having same Exits with TARGETKIND MFCC_E_D_A_Z

\begin{tabular}{|c|c|c|c|}
\hline Set ID & Scheme & $\%$ Corr & $\%$ Acc \\
\hline \multirow{3}{*}{ ES1 } & 1 & 95.83 & 98.61 \\
\hline & 2 & 95.8 & 98.61 \\
\hline & 3 & 91.67 & 97.22 \\
\hline \multirow{3}{*}{ ES2 } & 1 & 83.33 & 94.44 \\
\hline & 2 & 83.33 & 94.44 \\
\hline & 3 & 83.33 & 94.44 \\
\hline \multirow{3}{*}{ ES3 } & 1 & 100 & 100 \\
\hline & 2 & 100 & 100 \\
\hline & 3 & 100 & 100 \\
\hline \multirow{3}{*}{ ES4 } & 1 & 100 & 100 \\
\hline & 2 & 93.33 & 97.78 \\
\hline & 3 & 100 & 100 \\
\hline \multirow{3}{*}{ ES5 } & 1 & 100 & 100 \\
\hline & 2 & 100 & 100 \\
\hline & 3 & 100 & 100 \\
\hline \multirow{3}{*}{ ES6 } & 1 & 100 & 100 \\
\hline & 2 & 100 & 100 \\
\hline & 3 & 100 & 100 \\
\hline \multirow{3}{*}{ ES7 } & 1 & 83.33 & 94.44 \\
\hline & 2 & 83.33 & 94.44 \\
\hline & 3 & 100 & 100 \\
\hline \multirow{3}{*}{ ES8 } & 1 & 100 & 100 \\
\hline & 2 & 83.33 & 94.74 \\
\hline & 3 & 100 & 100 \\
\hline \multirow{3}{*}{ ES9 } & 1 & 75 & 92.31 \\
\hline & 2 & 75 & 92.31 \\
\hline & 3 & 75 & 92.31 \\
\hline \multirow{3}{*}{ ES10 } & 1 & 100 & 100 \\
\hline & 2 & 100 & 100 \\
\hline & 3 & 100 & 100 \\
\hline
\end{tabular}


MFCC E_D_A Z

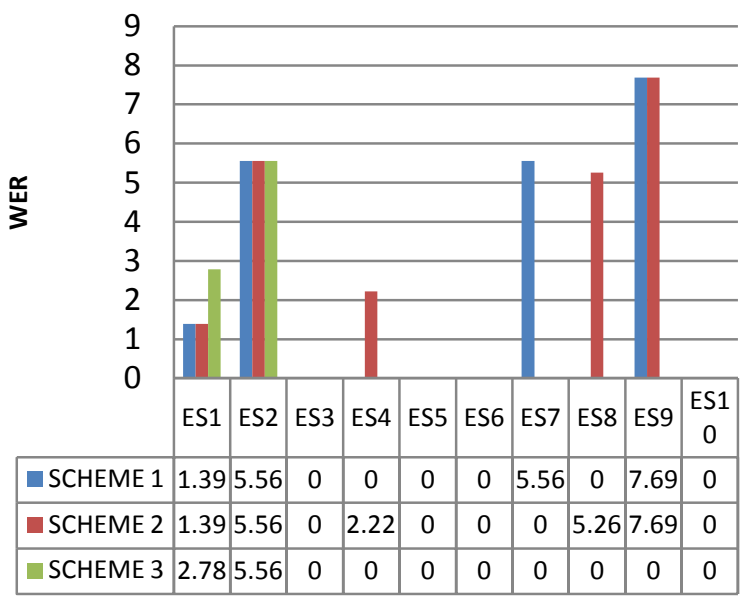

Fig 2: WER Results for Syllables having same Exits with TARGETKIND MFCC_E_D_A_Z

Results for Syllables having same characteristics with TARGETKIND "MFCC_0_D_A_Z" in Table 5 and Figure 3 .

Table 5. Speech Verification experiments results for Syllables having same Characteristics with

TARGETKIND MFCC_O_D_A_Z

\begin{tabular}{|c|c|c|c|}
\hline Set ID & Scheme & \% Corr & $\%$ Acc \\
\hline \multirow{3}{*}{$\mathrm{CS} 1$} & 1 & 92.11 & 97.37 \\
\hline & 2 & 86.84 & 95.61 \\
\hline & 3 & 97.37 & 99.12 \\
\hline \multirow{3}{*}{$\mathrm{CS} 2$} & 1 & 62.50 & 87.50 \\
\hline & 2 & 87.50 & 95.83 \\
\hline & 3 & 100 & 100 \\
\hline \multirow{3}{*}{$\mathrm{CS} 3$} & 1 & 96.67 & 98.89 \\
\hline & 2 & 87.50 & 92.71 \\
\hline & 3 & 80.56 & 91.67 \\
\hline \multirow{3}{*}{$\mathrm{CS} 4$} & 1 & 72.06 & 89.71 \\
\hline & 2 & 75.36 & 91.30 \\
\hline & 3 & 76.81 & 91.79 \\
\hline \multirow{3}{*}{ CS5 } & 1 & 93.75 & 97.92 \\
\hline & 2 & 100 & 100 \\
\hline & 3 & 100 & 100 \\
\hline \multirow{3}{*}{ CS6 } & 1 & 88.89 & 96.30 \\
\hline & 2 & 88.89 & 96.30 \\
\hline & 3 & 88.89 & 96.30 \\
\hline \multirow{3}{*}{ CS7 } & 1 & 82.61 & 94.20 \\
\hline & 2 & 84.06 & 94.69 \\
\hline & 3 & 85.51 & 95.17 \\
\hline \multirow{3}{*}{ CS8 } & 1 & 100 & 100 \\
\hline & 2 & 100 & 100 \\
\hline & 3 & 100 & 100 \\
\hline \multirow{3}{*}{ CS9 } & 1 & 88.89 & 96.30 \\
\hline & 2 & 94.44 & 98.15 \\
\hline & 3 & 100 & 100 \\
\hline \multirow{3}{*}{ CS10 } & 1 & 81.48 & 93.83 \\
\hline & 2 & 77.78 & 92.59 \\
\hline & 3 & 75.93 & 91.98 \\
\hline
\end{tabular}

MFCC_O_D_A_Z

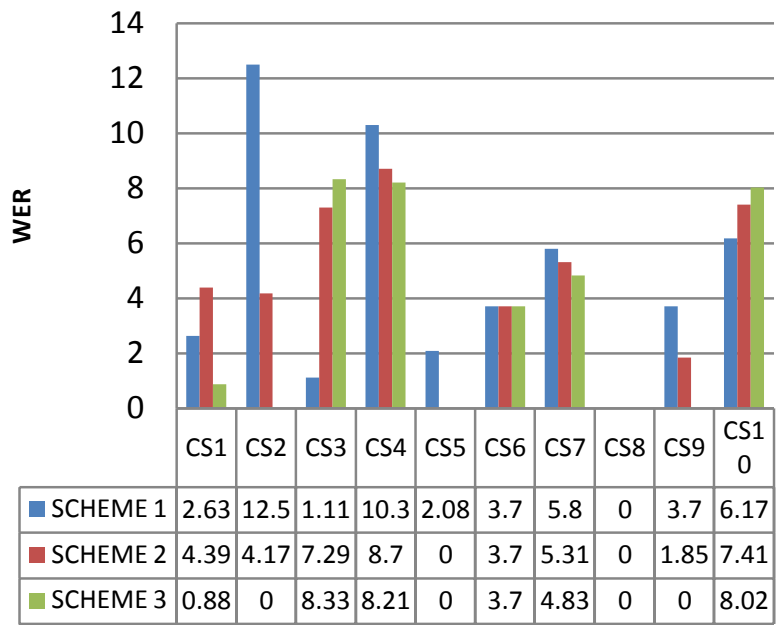

Fig 3: WER Results for Syllables having same Characteristics with TARGETKIND MFCC_O_D_A_Z

Results for Syllables having similar characteristics with TARGETKIND "MFCC_E_D_A_Z" in Table 6 and Figure 4.

Table 6. Speech Verification experiments results for Syllables having same Characteristics with

TARGETKIND MFCC_E_D_A_Z

\begin{tabular}{|c|c|c|c|}
\hline Set ID & Scheme & $\%$ Corr & \%Асc \\
\hline \multirow{3}{*}{$\mathrm{CS} 1$} & 1 & 97.37 & 99.12 \\
\hline & 2 & 100 & 100 \\
\hline & 3 & 100 & 100 \\
\hline \multirow{3}{*}{$\mathrm{CS} 2$} & 1 & 87.50 & 95.83 \\
\hline & 2 & 100 & 100 \\
\hline & 3 & 100 & 100 \\
\hline \multirow{3}{*}{ CS3 } & 1 & 93.33 & 97.78 \\
\hline & 2 & 95.65 & 98.55 \\
\hline & 3 & 90.70 & 96.90 \\
\hline \multirow{3}{*}{ CS4 } & 1 & 69.57 & 89.37 \\
\hline & 2 & 70.42 & 90.14 \\
\hline & 3 & 74.65 & 91.55 \\
\hline \multirow{3}{*}{ CS5 } & 1 & 93.75 & 97.92 \\
\hline & 2 & 93.75 & 97.92 \\
\hline & 3 & 93.75 & 97.92 \\
\hline \multirow{3}{*}{ CS6 } & 1 & 100 & 100 \\
\hline & 2 & 100 & 100 \\
\hline & 3 & 100 & 100 \\
\hline \multirow{3}{*}{ CS7 } & 1 & 94.20 & 98.07 \\
\hline & 2 & 91.18 & 97.06 \\
\hline & 3 & 85.29 & 94.61 \\
\hline \multirow{3}{*}{ CS8 } & 1 & 83.33 & 94.44 \\
\hline & 2 & 83.33 & 94.44 \\
\hline & 3 & 83.33 & 94.44 \\
\hline \multirow{3}{*}{ CS9 } & 1 & 94.12 & 98.04 \\
\hline & 2 & 100 & 100 \\
\hline & 3 & 100 & 100 \\
\hline \multirow{3}{*}{ CS10 } & 1 & 85.19 & 94.44 \\
\hline & 2 & 85.19 & 95.06 \\
\hline & 3 & 83.33 & 94.44 \\
\hline
\end{tabular}




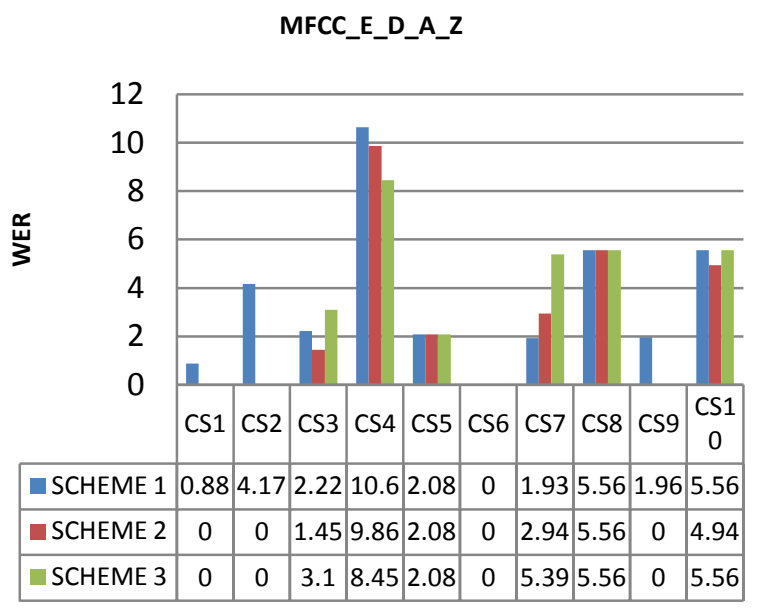

Fig 4: WER Results for Syllables having same Characteristics with TARGETKIND MFCC_E_D_A_Z

\section{CONCLUSION}

In this paper comparative evaluation of various MFCC implementations was performed using HTK Toolkit on speaker independent speech. Speech recognizer was tested experimentally with different feature sets; Each of syllables sets have different model for best Accuracy results.

As expected, the verification performance did not vary vastly with changing schemes for syllables having same exits, However, some of these sets which have common characteristics beside (as in set ES1 "TTAA, DAAL, TAA") have shown observable performance change.

On the other hand; syllables sets having same characteristics can show clear change in performance with different schemes; specially with sets that have some common syllables (as for sets containing BAA, or TTAA such as CS2 and CS3 ).

Overall, our experiments showed that WER varies between 0 and 10 for syllables exits sets and between 0 and 12.5 for characteristics sets.

The work implemented in this paper is a step towards the development of a complete Automatic Verification system for Classical Arabic (CA).

Although speech recognition's accuracy is expected to increase as the training corpus increase. Our training corpus is limited to different instants of the professional readers to detect fine changes between the trainee and the trainer.

\section{REFERENCES}

[1] http://www.al-bab.com/arab/language/lang.htm

[2] http://en.wikipedia.org/wiki/Arabic_language

[3] Nour Mohamed Hakany, " القاعدة النورانية بالتوجيهات "الفتحية"

[4] Ibrahim AbdelMohsen El Bedewy, Mohamed Abed El Qarshy, " الأثر العلمي لتدريس قاعدة النور في حلقات تحفيظ . King Abdul Aziz University.
[5] Mohamed Elmahdy, Rainer Gruhn, and Wolfgang Minker, "Novel Techniques for Dialectal Arabic Speech Recognition”, Springer, pp. 2-4, 2012.

[6] Zaidi Razak, Noor Jamaliah Ibrahim, Emran Mohd Tamil, Mohd Yamani Idna Idris, Mohd. Yakub and ZulkifliBin Mohd Yusoff, "Quranic Verse Recitation Feature Extraction Using Mel-Frequency Cepstral Coefficient(MFCC)", In Proceedings of the 4th IEEE International Colloquium on Signal Processing and its Application(CSPA) 2008, 7-9 March 2008, Kuala Lumpur, MALAYSIA.

[7] H. Tabbal, et al "Analysis and Implementation of an Automated Delimiter of Qur'anic Verses in Audio Files using Speech Recognition Techniques". Chapter of the Book "Robust Speech Recognition and Understanding", edited by: Michael Grimm and Kristian Kroschel, 2007.

[8] Bassam A. Q. Al-Qatab , Raja N. Ainon, "Arabic Speech Recognition Using Hidden Markov Model Toolkit(HTK)", 978-1-4244-6716-711 0/\$26.00 @2010 IEEE.

[9] Vimala.C and Dr.V.Radha, "A Review on Speech Recognition Challenges and approaches", World of Computer Science and Information Technology Journal (WCSIT) ISSN: 2221-0741 Vol. 2, No. 1, 1-7, 2012.

[10] Yacine Yekache, Yekhlef Mekelleche, Belkacem Kouninef, "Towards Quranic reader controlled by speech", (IJACSA) International Journal of Advanced Computer Science and Applications, Vol. 2, No. 11, 2011.

[11] Sunita Shah, Tanvi Shah "Arabic a profile" , London SIG Bilingualism, 2007.

[12] S. Young, G. Evermann, M. Gales, T. Hain, D. Kershaw, X. Liu, G. Moore, J. Odell, D. Ollason, D. Povey, J. Odell, D. Ollason, D. Povey, V. Valtchev, and P. Woodland, "The HTK Book. Version 3.4", Department of Engineering, Cambridge University, UK, 2009.

[13] htp://www.voxforge.org/home/forums/messageboards/acoustic-model-discussions/htk-mfcc-study (http://mphmedia.net/mfccStudy.html)

[14] K. Kumar and R. K. Aggarwal "Hindi Speech Recognition System using HTK" International journal of Computing and Business Research ISSN Vol. 2 issue 2 May 2011.

[15] HTK "Hidden Markov Model Toolkit", available at http://htk.eng.cam.ac.uk,2012.

[16] Mohit Dua, R.K.Aggarwal, Virender Kadyan and Shelza Dua, "Punjabi Automatic Speech Recognition Using HTK", IJCSI International Journal of Computer Science Issues, vol. 9, issue 4, no. 1, July 2012.

[17] Ryan, M.S. and Nudd, G.R.: The Viterbi Algorithm. Department of Computer Science, University of Warwick, Coventry, CV4 7AL, England, February, 1993. 